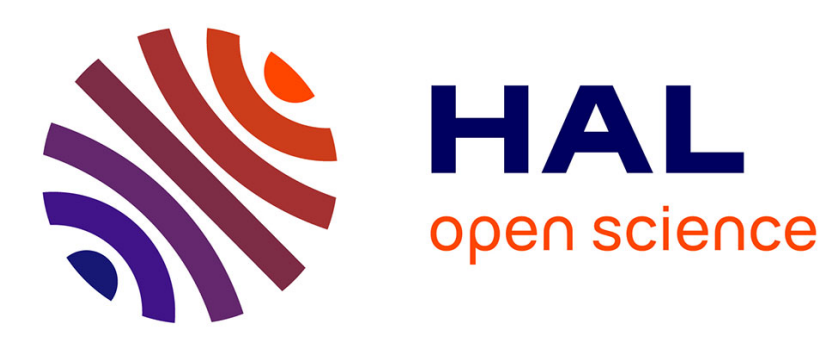

\title{
Pharmaceutical silver doped clays : an EXAFS study from silver to silicon K-edges absorption
}

\author{
F. Keller-Besrest, S. Benazeth, Ch. Souleau
}

\section{To cite this version:}

F. Keller-Besrest, S. Benazeth, Ch. Souleau. Pharmaceutical silver doped clays: an EXAFS study from silver to silicon K-edges absorption. Journal de Physique IV Proceedings, 1994, 04 (C9), pp.C9299-C9-302. 10.1051/.jp4:1994952 . jpa-00253514

\section{HAL Id: jpa-00253514 https://hal.science/jpa-00253514}

Submitted on 1 Jan 1994

HAL is a multi-disciplinary open access archive for the deposit and dissemination of scientific research documents, whether they are published or not. The documents may come from teaching and research institutions in France or abroad, or from public or private research centers.
L'archive ouverte pluridisciplinaire HAL, est destinée au dépôt et à la diffusion de documents scientifiques de niveau recherche, publiés ou non, émanant des établissements d'enseignement et de recherche français ou étrangers, des laboratoires publics ou privés. 


\title{
Pharmaceutical silver doped clays: an EXAFS study from silver to silicon K-edges absorption
}

\author{
F. Keller-Besrest, S. Benazeth**** and Ch. Souleau*
}

Laboratoire de Biomathématiques et Physique, UFR de Pharmacie, Université Paris V, René Descartes, 4 Avenue de l'Observatoire, 75270 Paris, France

* Laboratoire de Chimie-Physique Minérale et Bioinorganique, Faculté de Pharmacie, Université Paris XI, 92296 Châtenay Malabry, France

** LURE, Bât. 209D, Centre Universitaire d'Orsay, 91405 Orsay, France

\begin{abstract}
.
The remarkable adsorption properties of the montmorillonite clay are used to elaborate a silver doped clay, intented to a therapeutical application to burns. The unstable properties of the material obtained lead us to examine the localization of the added silver in the clay matrix by EXAFS spectroscopy performed at the Ag and Si K-edge. The results show that Ag atoms formed in triangular clusters are coordinated to the triangular designs of oxygen atoms on the border plane of the layers of the clay. The local negative charge in the border plane explains the reduction of $\mathrm{Ag}^{+}$ ions, at the origin of the unstability observed for this material.
\end{abstract}

\section{1 - INTRODUCTION.}

The remarkable adsorption properties of the montmorillonite clay (smectite group) originate from its structure built of twodimensional tetrahedral sheets linked by one octahedral sheet, forming a T-O-T layer, the interlayer space presenting various thicknesses. (Fig 1). The $\mathrm{SiO}_{4}$ tetrahedra share three oxygen corners (the basal oxygens) that form an hexagonal mesh pattern on the border of the TO-T layer. Isomorphic substitutions of lesscharged cations in the $\mathrm{Al}(\mathrm{O}, \mathrm{OH}) 6$ octahedral sheet produce a resultant negative charge on the layers. Individual or hydrated cations $\left(\mathrm{Na}^{+}\right.$, $\left.\mathrm{K}+, \mathrm{Mg}^{2+} \ldots.\right)$ which are present in the interlayer space, balance this apparent negative charge. In the montmorillonite, the low density charge in the layer border plane, and the soft bonding with the interlayer cations explain the possible exchange with other species.

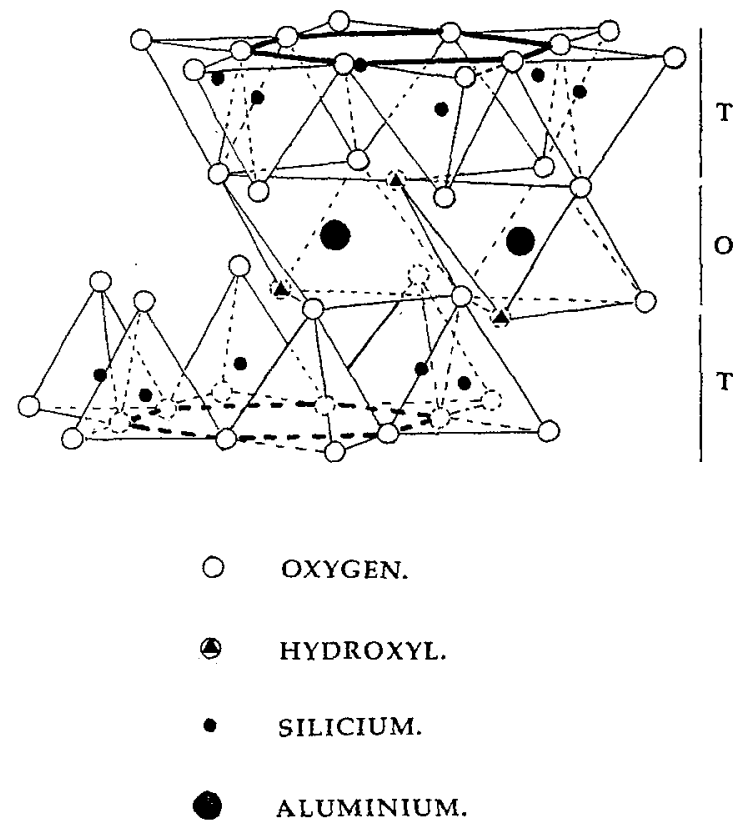

FIG 1 : Atomic structure of a layer of montmorillonite. 
The $\mathrm{Ag}^{+}$added clay is prepared by contact with a $\mathrm{AgNO}_{3}$ solution, and the capacity of fixation presents a saturation level of about $7,7 \mathrm{mg}$ per gramme of clay. material.

EXAFS spectroscopy at the Ag and Si K-edge is performed to study the structure of this poorly

\section{2 - EXAFS}

\section{RESULTS.}

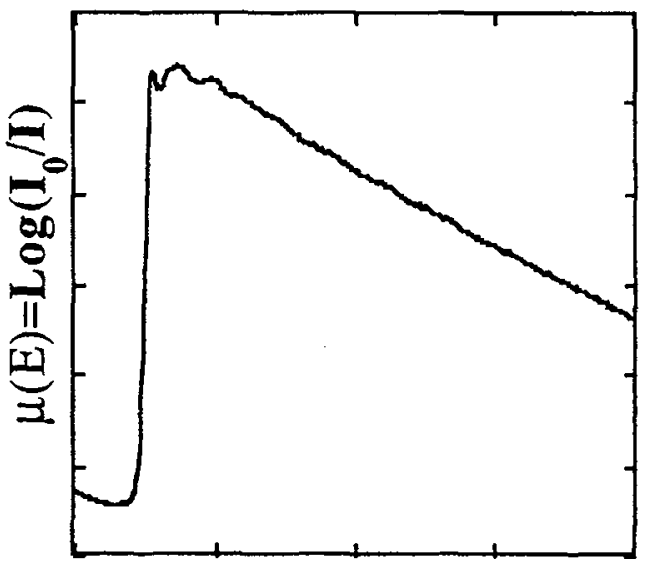

$25400 \quad 25800 \quad 26200 \mathrm{E}(\mathrm{eV})$

FIG 2 : Absorption spectrum collected at the $\mathrm{Ag} \mathrm{K}$-edge.

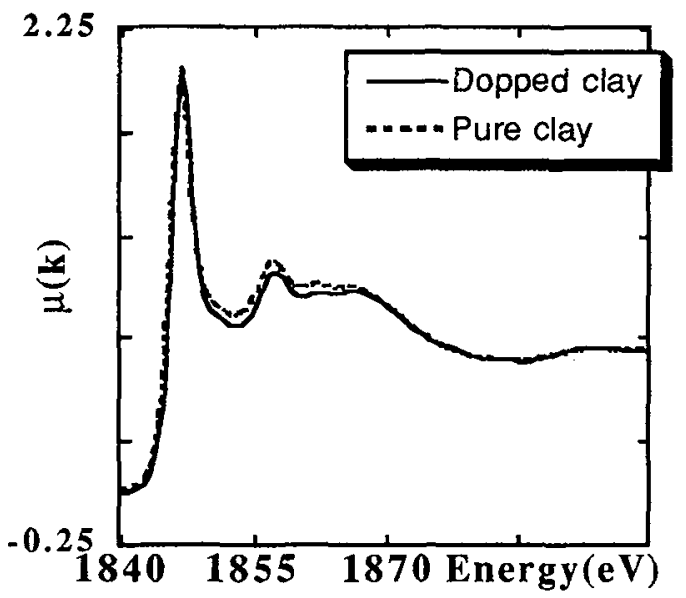

We have used EXAFS technique at two extremal energy ranges as we need to register $\mathrm{X}$-Ray absorption spectra around $\mathrm{Ag} \mathrm{K}$-edge $(25520 \mathrm{eV})$ at DCI (storage ring Orsay), and around Si K-edge $(1848 \mathrm{eV})$ at Super Aco (Orsay). Thus, the sample preparation was adapted to these hard and soft X-Rays and, the spectra resolution, the various features were observed at different levels.

Concerning the Ag K-edge, the low content ( 8 $\mathrm{mg} / \mathrm{g}$ ) of silver obliged us to prepare thick pellets (about $1 \mathrm{~cm}$ ), and the spectra were collected at liquid He temperature to limit the thermal disorder of $\mathrm{Ag}$ atoms. The spectra present weak oscillations related to silver dilution. (Fig 2) Because of the weakness of the signal, it was rather difficult to obtain the normalized EXAFS spectra. The interpretation of the radial distribution function in relation with the structural problem investigated was not easy: either the interlayer $\mathrm{Ag}^{+}$cations are trapped in the hexagonal cavities in the border plane of the layer, and so are neighboured by one $\mathrm{Si}$ atom of the $\mathrm{T}$ sheet, or they are bounded in a different configuration to $\mathrm{O}$ atoms of the border plane.

Two series of XANES and absorption spectra of samples of pure and $\mathrm{Ag}^{+}$-dopped clay are collected at the Si K-edge. The samples of about $1 \mathrm{~mm}$ thickness are prepared by decantation on a Micropore film. Figure 3 shows that the spectra are quite identical, which rules out the hypothesis of a $\mathrm{Ag}$-Si bounding.

A final treatment of the data lead to a RDF which can be interpreted: a linear model describes the pre-edge backgound, and the post-edge absorption is reproduced by a fifth degree polynomial function. Fourier transform using an Hamming window on a selected part of the normalized EXAFS spectra $(2.6<k<13,6$ $\AA^{-1}$ ) gives the RDF in figure 4 where the peaks ending to the final fits are indicated.

Fig 3 : XANES spectra at the Si K-edge. 
The fits are obtained using experimental phase and amplitude backscattering functions extracted from Ag2O and $\mathrm{Ag}$ metal reference compounds treated in the same way as the samples studied. Table 1 presents the final parameters obtained, and figure $5 \mathrm{a}$ and $\mathrm{b}$ the fits of the two shells identified. They describe the $\mathrm{Ag}$ atoms surrounding as made of a first shell of $\mathrm{O}$ atoms, and a second one of $\mathrm{Ag}$ atoms. The Debye-Waller factors $\Delta \sigma$ are the difference between thermal disorder terms of $\mathrm{O}$ and $\mathrm{Ag}$ atoms in the crystalline reference compounds and the much less organized doped clay.

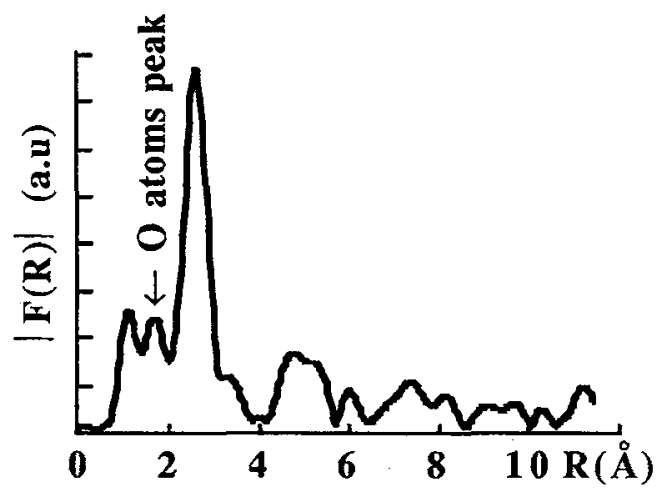

FIG 4 : RDF function at the Ag K-edge.

The major peak corresponds

to the $\mathrm{Ag}$ atoms second shell.

TABLE 1: Silver surrounding atoms.Parameters obtained from the fits.

$\mathrm{N}$ : number of coordination, $\mathrm{R}$ : Ag-atom distance, $\Delta \sigma$ : Debye-Waller factor.

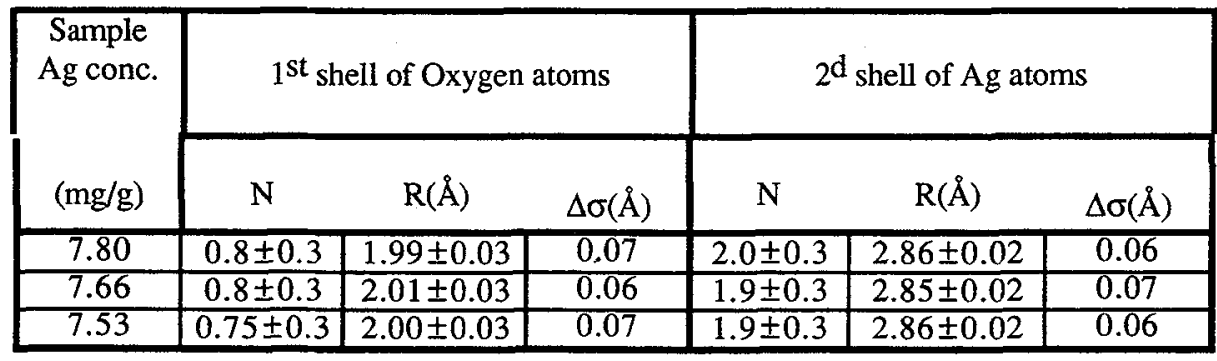

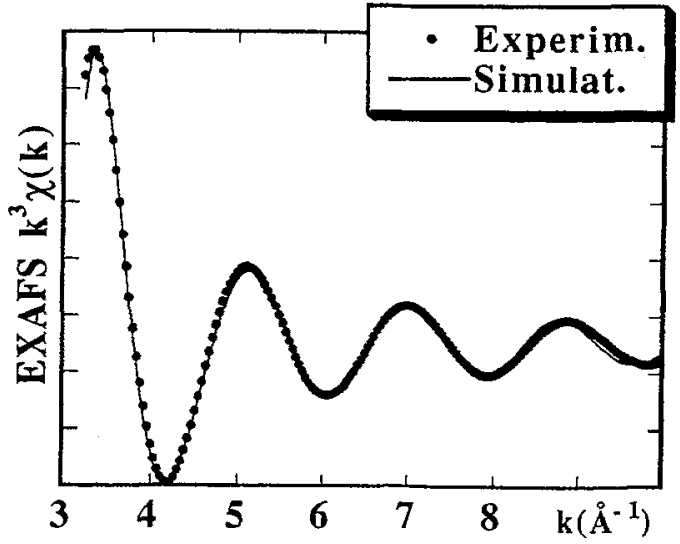

FIG 5 a : Fit of the first peak (O atoms shell)

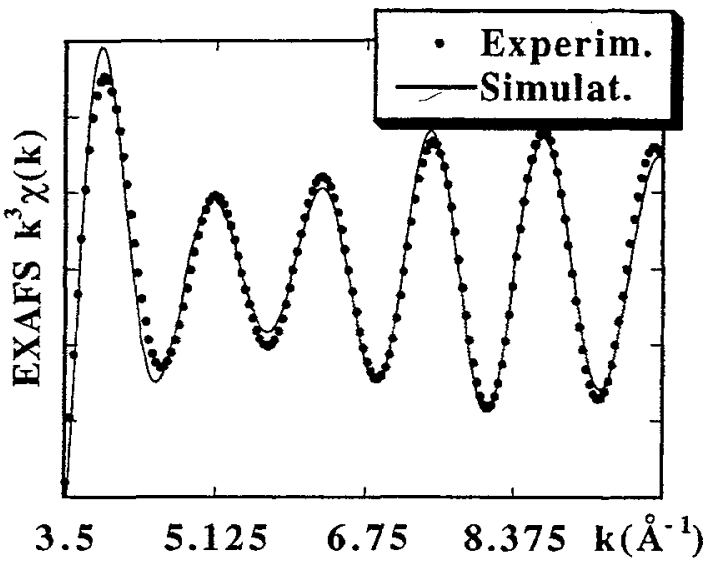

FIG 5 bit of the second peak (Ag atoms shell) 


\section{3 - DISCUSSION.}

The environment of each silver added atom is made of two silver atoms at a distance of $2,86 \AA$, which is close to the Ag-Ag distance in metal $(2,89 \AA)$ and for each one, as a mean, in the order of 0.8 neighbour oxygen atom. These results rule out the formation of silver oxide $\mathrm{Ag}_{2} \mathrm{O}$. The figure which interprets these structural results is an aggregation of the additional silver atoms in triangular clusters. The tendancy of $\mathrm{Ag}^{+}$cations to form such triangular motifs is known in ternary silver oxides and some other inorganic silver compounds $(1,2,3)$.

On the border plane of the T-O-T layers of the clay, oxygen atoms form a pattern of hexagonal cavities, linked by oxygen triangular figures which are the base of the $\mathrm{SiO}_{4}$ tetrahedra. Because of the isomorphic substitution of lesscharged cations in the octahedra (mainly $\mathrm{Mg}^{2+}$ substituted to $\left.\mathrm{Al}^{3+}\right)$, some oxygen atoms bear a partial negative charge which can act as a fixation point of the $\mathrm{Ag}^{+}$added cations. According to the localization of these negative charges in the triangular figures of oxygen atoms, one, two, or each of the three atoms of the silver clusters are bounded to oxygen atoms (Fig 6). The total mean number of oxygen neighbours (in the order of 0.8 ) results from the sum of the mean number of oxygen neigbours for each silver atom $(1 / 3,2 / 3,1)$, weighted by the occurence ratio of each of the three configurations, which cannot be estimated by our structural investigation. This accounts for the results of EXAFS analysis: the $\mathrm{Ag}-\mathrm{Ag}$ distance in the clusters $(2,86 \AA)$ is compatible with the O.O distance $(2,60 \AA)$ on the basis of the $\mathrm{SiO}_{4}$ tetrahedra, and the Ag-O distance $(2,00 \AA)$ between a silver atom and an oxygen atom of basal plane is close to the $\mathrm{Ag}-\mathrm{O}$ distance $(2,06 \AA)$ in $\mathrm{Ag}_{2} \mathrm{O}$.

Recently, the study of the redox reactions in the photographic process pointed out the fundamental part assumed by the formation of clusters of silver atoms (4). In the photographic emulsion, after the reduction reaction $\mathrm{Ag}^{+}+\mathrm{e}$ $\Rightarrow \mathrm{Ag}^{0}$, silver atoms gather and form clusters which are surrounded by $\mathrm{Ag}^{+}$ions which form a complex like pair $\mathrm{Ag}^{0}$. $\mathrm{Ag}^{+}$, in which these two atoms share a positive charge. On the border plane of the layers, some oxygen atoms are fixation points, and donors of electrons which lead to the reduction of one, two or three of the bounded $\mathrm{Ag}^{+}$ions of the clusters, which then contain either the two species $\mathrm{Ag}^{\circ} / \mathrm{Ag}^{+}$, or only $\mathrm{Ag}^{\circ}$ atoms.

Our model of Ag clusters in the clay is obtained from experimental data collected at $4.2 \mathrm{~K}$, because the high thermal disorder of $\mathrm{Ag}$ atoms prevents to register a good quality absorption spectra at RT. However our model can be extrapolated at RT where the existence of such clusters in a higher thermal energy state is demonstrated in the photographic process previously referenced.

According to this model, the formation of metallic silver clusters results from the interaction of $\mathrm{Ag}^{+}$ions with the clay which acts like a donor of electron medium.(The high Debye-Waller factor of oxygen surrounding atoms (Table 1) indicates a soft bounding between the clusters and the border plane). This description explains the blackening of the silver added clay and the difficulty to extract further a regular concentration of the $\mathrm{Ag}^{+}$ ions trapped in the clusters.

* Thanks to Beaufour-Ipsen Laboratory for providing the samples of clay, and to A.M.Flank for assistance during Super Aco experiments.

\section{References:}

1) JANSEN, M. , J. Less-Common Met. 76 (1980) 285.

2) JANSEN, M. Angew. Chem. Int. Ed. Engl. 26 (1987) 1098-1110.

3) HAMILTON, J . F . Advances in Physics . $37 \mathrm{~N}^{\circ} 4$ (1988) p 359-441

4) BELLONI-COFIER, J., La Recherche No217 21, (janvier 1990) p 50-56 